Moroccan J. of Pure and Appl. Anal. (MJPAA)

Volume 8(1), 2022, Pages 44-53

ISSN: Online 2351-8227 - Print 2605-6364

DOI: $10.2478 / \mathrm{mjpaa}-2022-0004$

\title{
Existence and multiplicity results for a Steklov problem involving $(p(x), q(x))$-Laplacian operator
}

\author{
BELHADJ KARIM ${ }^{1}$, A. LAKHDI ${ }^{1}$, M. R. SIDI AMMI², A. ZEROUALI ${ }^{3}$
}

AвstRACT. In this work, we are concerned with a generalized Steklov problem with $(p(x), q(x))$-Laplacian operator. Under some appropriate conditions on the data involved in the elliptic problem, we prove the existence of at least three solutions using Ricceri's three critical points theorem.

Mathematics Subject Classification (2020). 35J65, 35J60, 47J30, 58E05.

Key words and phrases. Variable exponents, Variational methods, Critical points theorem.

\section{Introduction and main results}

The study of variational problems and elliptic equations with variable exponent has been received considerable attention in recent years. This is due to the fact that they have many applications to a various range of phenomena including elastic mechanics[33], electro-rheological and thermo-rheological viscous flows of non-Newtonian fluids[3], image restoration[11] and mathematical biology[17]. In major nonhomogeneous materials, the classical approach based on Lebesgue and Sobolev spaces seems to be not suitable. This implies the growing interest of

Received December 8, 2020 - Accepted May 08, 2021.

(C) The Author(s) 2021. This article is published with open access by Sidi Mohamed Ben Abdallah University.

${ }^{1}$ University Moulay Ismail of Meknes, FST Errachidia, LMIMA Laboratory, ROLALI Group, Morocco.

e-mail: karembelf@gmail.com, lakhdi.abdessamad@gmail.com

${ }^{2}$ University Moulay Ismail of Meknes, FST Errachidia, MAIS Laboratory, AMNEA Group, Morocco.

e-mail: rachidsidiammi@yahoo.fr (Corresponding Author)

${ }^{3}$ Regional Centre of Trades Education and Training, Oujda, Morocco.

e-mail: abdellahzerouali@yahoo.fr. 
researchers for the study of variable exponent Lebesgue and Sobolev spaces. The paper of Orlicz [21] was the first spark that gave birth to works in relation to variable exponent Lebesgue spaces. We point out that several physical models are describing by equations involving $p(x)$ growth condition [1]. Owing to its non homogeneity, the $p(x)$-Laplacian operator where the real-valued function $p($.$) is a continuous function presents more complicated properties than$ the $p$-Laplacian operator. Reference [13] represents one of the most important collections of results regarding the analysis of variable exponent Lebesgue and Sobolev spaces. For more applications of variable exponent equations, we cite the references $[4,5,6,22,25,26]$. Important contributions of existence, nonexistence and properties of solutions for variable exponent problems can be found in $[12,14,27,28,29]$. On the other hand, Ricceri's three critical points theorem is a powerful tool to study boundary problem of differential equation (see, $[2,7,8,9]$ ). Many authors studied the case where $p(x)=q(x)$ with Dirichlet, Neumann or Steklov boundary conditions on a bounded domain (see for example, [19, 20, 30, 32] ). In particularly, A. Zerouali et al [32] used three critical points theorem of Ricceri to study the following elliptic problem with nonlinear boundary conditions and variable exponent

$$
\begin{array}{r}
-\operatorname{div}[a(x, \nabla u)]+|u|^{p(x)-2} u=\lambda f(x, u) \quad \text { in } \Omega, \\
a(x, \nabla u) \cdot v=\mu g(x, u) \quad \text { on } \partial \Omega .
\end{array}
$$

They proved existence of three solutions for the problem (1.1) under appropriate hypotheses. Our main, in this paper, is to extend the results of the $p(x)$-Laplacian given in [32] to $(p(x), q(x))$-Laplacian.

Let $\Omega$ be a bounded domain in $\mathbb{R}^{N}(N \geq 2)$ with smooth boundary $\partial \Omega$, and $p, q \in C(\bar{\Omega})$ such that $q(x) \leq p(x)$ for all $x \in \bar{\Omega}$. We consider the problem

$$
\begin{cases}\triangle_{p(x)} u+\triangle_{q(x)} u=|u|^{p(x)-2} u+|u|^{q(x)-2} u-\lambda f(x, u) & \text { in } \Omega, \\ \left(|\nabla u|^{p(x)-2}+|\nabla u|^{q(x)-2}\right) \frac{\partial u}{\partial v}=\mu g(x, u) & \text { on } \partial \Omega,\end{cases}
$$

where $\lambda, \mu \in[0, \infty)$ and $v$ is the outward unit normal vector on $\partial \Omega$. $f: \Omega \times \mathbb{R} \mapsto \mathbb{R}$ and $g: \partial \Omega \times \mathbb{R} \mapsto \mathbb{R}$ are two Carathéodory functions.

We denote

$$
\begin{gathered}
h^{-}=\min _{x \in \bar{\Omega}} h(x) ; \quad h^{+}=\max _{x \in \bar{\Omega}} h(x) ; \quad \text { for all } h \in C(\bar{\Omega}), \\
p^{*}(x)= \begin{cases}\frac{N p(x)}{N-p(x)} & \text { if } p(x)<N, \\
+\infty & \text { if } p(x) \geq N,\end{cases} \\
p^{\partial}(x)= \begin{cases}\frac{(N-1) p(x)}{N-p(x)} & \text { if } p(x)<N, \\
+\infty & \text { if } p(x) \geq N,\end{cases}
\end{gathered}
$$

and

$$
C_{+}(\bar{\Omega})=\left\{h \in C(\bar{\Omega}): 1<h^{-}<h^{+}<+\infty\right\} .
$$

We assume that variable exponents $p$ and $q$ are in $C_{+}(\bar{\Omega})$.

The energy functional corresponding to problem (1.2) is defined on $W^{1, p(x)}(\Omega)$ as

$$
H(u)=\Phi(u)+\lambda \Psi(u)+\mu J(u),
$$


where

$$
\begin{aligned}
\Phi(u) & =\int_{\Omega}\left(\frac{|\nabla u|^{p(x)}}{p(x)}+\frac{|\nabla u|^{q(x)}}{q(x)}\right) d x+\int_{\Omega}\left(\frac{|u|^{p(x)}}{p(x)}+\frac{|u|^{q(x)}}{q(x)}\right) d x, \\
\Psi(u) & =-\int_{\Omega} F(x, u) d x, \\
J(u) & =-\int_{\partial \Omega} G(x, u) d \sigma,
\end{aligned}
$$

with $F(x, u)=\int_{0}^{u} f(x, s) d s, G(x, u)=\int_{0}^{u} g(x, s) d s$ and $d \sigma$ is the $N-1$ dimensional Hausdorff measure. Let us recall that $u \in W^{1, p(x)}(\Omega)$ is a weak solution of (1.2) if

$$
\begin{aligned}
& \int_{\Omega}\left(|\nabla u|^{p(x)-2}+|\nabla u|^{q(x)-2}\right) \nabla u \nabla v d x+\int_{\Omega}\left(|u|^{p(x)-2}+|u|^{q(x)-2}\right) u v d x \\
& =\lambda \int_{\Omega} f(x, u) v d x+\mu \int_{\partial \Omega} g(x, u) v d \sigma, \quad \text { for all } v \in W^{1, p(x)}(\Omega) .
\end{aligned}
$$

We now describe the hypotheses of the functions $f, F$ and $g$ as follows

(H1) For $t \in C(\bar{\Omega})$ and $t(x)<p^{*}(x)$ for all $x \in \bar{\Omega}$, we have

$$
\sup _{(x, s) \in \Omega \times \mathbb{R}} \frac{|f(x, s)|}{1+|s|^{t(x)-1}}<+\infty ;
$$

(H2) There exists positive constant $c_{1}$ such that $F(x, s)>0$ for a.e. $x \in \Omega$ and all $\left.\left.s \in\right] 0, c_{1}\right]$;

(H3) There exists $p_{1}(x) \in C(\bar{\Omega})$ and $p^{+}<p_{1}^{-} \leq p_{1}(x)<p^{*}(x)$, such that

$$
\limsup _{s \rightarrow 0} \sup _{x \in \Omega} \frac{F(x, s)}{|s|^{p_{1}(x)}}<+\infty ;
$$

(H4) There exist positive constant $c_{2}$ and a function $\gamma(x) \in C(\bar{\Omega})$ with $1<\gamma^{-} \leq \gamma^{+}<p^{-}$, such that $|F(x, s)| \leq c_{2}\left(1+|s|^{\gamma(x)}\right)$ for a.e. $x \in \Omega$ and all $s \in \mathbb{R}$;

(H5) For $p_{2}(x) \in C(\bar{\Omega})$ and $p_{2}(x)<p^{\partial}(x)$ for all $x \in \bar{\Omega}$, we have

$$
\sup _{(x, s) \in \partial \Omega \times \mathbb{R}} \frac{|g(x, s)|}{1+|s|^{p_{2}(x)-1}}<+\infty .
$$

Example 1.1. Let $r, s \in C_{+}(\bar{\Omega})$. If $r^{+}, s^{+}<p^{-}$, then the function $f(x, u)=|u|^{r(x)-2} u$ verifies the hypotheses (H1)-(H4) and the function $g(x, u)=|u|^{s(x)-2} u$ satisfies (H5).

The main result of this paper is the following theorem.

Theorem 1.1. Assume $(H 1)-(H 4)$. Then there exist an open interval $\Lambda \subseteq(0,+\infty)$ and a positive real number $\omega$ such that, for each $\lambda \in \Lambda$ and each function $g: \partial \Omega \times \mathbb{R} \rightarrow \mathbb{R}$ satisfying (H5), there exists $\delta>0$ which satisfies, for each $\mu \in[0, \delta]$, the problem (1.2) has at least three weak solutions whose norms in $W^{1, p(x)}(\Omega)$ are less than $\omega$.

The paper is organized into three sections. The first Section contains an introduction and the main result. In the second Section, we recall some basic and elementary properties concerning the generalized Lebesgue-Sobolev spaces. We further recall Biagio-Ricceri's three critical points 
theorem (Theorem 2.1), which is the key tool of our proof. The third Section is devoted for the proof of our main result.

\section{Preliminaries}

We first recall some background facts concerning the generalized Lebesgue-Sobolev spaces. For $p \in C_{+}(\bar{\Omega})$, we introduce the variable exponent Lebesgue space

$$
L^{p(x)}(\Omega):=\left\{u: \Omega \subset \mathbb{R}^{N} \rightarrow \mathbb{R} \text { is measurable and } \int_{\Omega}|u|^{p(x)} d x<+\infty\right\},
$$

endowed with the Luxemburg norm

$$
|u|_{L^{p(x)}(\Omega)}:=\inf \left\{\alpha>0 ; \int_{\Omega}\left|\frac{u(x)}{\alpha}\right|^{p(x)} d x \leq 1\right\},
$$

which is separable and reflexive Banach space (see, [16]). Let us define the space

$$
W^{1, p(x)}(\Omega):=\left\{u \in L^{p(x)}(\Omega) /|\nabla u| \in L^{p(x)}(\Omega)\right\},
$$

equipped with the norm

$$
\|u\|:=\inf \left\{\alpha>0 ; \int_{\Omega}\left|\frac{u(x)}{\alpha}\right|^{p(x)} d x+\int_{\Omega}\left|\frac{\nabla u(x)}{\alpha}\right|^{p(x)} d x \leq 1\right\} ; \quad \forall u \in W^{1, p(x)}(\Omega) .
$$

\section{Proposition 2.1. $[14,18]$}

(1) $W^{1, p(x)}(\Omega)$ is separable reflexive Banach space;

(2) If $h \in C_{+}(\bar{\Omega})$ and $h(x)<p^{*}(x)$ for any $x \in \bar{\Omega}$, then the embedding from $W^{1, p(x)}(\Omega)$ to $L^{h(x)}(\Omega)$ is compact and continuous.

(3) If $h \in C_{+}(\bar{\Omega})$ and $h(x)<p^{\partial}(x)$ for any $x \in \bar{\Omega}$, then the embedding from $W^{1, p(x)}(\Omega)$ to $L^{h(x)}(\partial \Omega)$ is compact and continuous.

An important role in manipulating the generalized Lebesgue-Sobolev spaces is played by the mapping $\rho_{p}$ defined by

$$
\rho_{p}(u):=\int_{\Omega}|\nabla u|^{p(x)} d x+\int_{\Omega}|u|^{p(x)} d x, \quad \forall u \in W^{1, p(x)}(\Omega) .
$$

Proposition 2.2. ([12]) For $u, u_{k} \in W^{1, p(x)}(\Omega) ; \quad k=1,2, \ldots$, we have

(1) $\|u\| \geq 1$ implies $\|u\|\left\|^{p^{-}} \leq \rho_{p}(u) \leq\right\| u \|^{p^{+}}$;

(2) $\|u\| \leq 1$ implies $\|u\|\left\|^{p^{+}} \leq \rho_{p}(u) \leq\right\| u \|^{p^{-}}$;

(3) $\left\|u_{k}\right\| \rightarrow 0$ as $k \rightarrow+\infty$ if and only if $\rho_{p}\left(u_{k}\right) \rightarrow 0$ as $k \rightarrow+\infty$;

(4) $\left\|u_{k}\right\| \rightarrow+\infty$ as $k \rightarrow+\infty$ if and only if $\rho_{p}\left(u_{k}\right) \rightarrow+\infty$ as $k \rightarrow+\infty$.

In order that the paper be more complete, we recall the revised form of Ricceri's three critical points theorem [24, Theorem 1] and [23, Proposition 3.1]. 
Theorem 2.1. [24, Theorem 1]Let $X$ be a reflexive real Banach space. $\Phi: X \rightarrow \mathbb{R}$ is a continuously Gâteaux differentiable and sequentially weakly lower semicontinuous functional whose Gâteaux derivative admits a continuous inverse on $X^{\prime}$, where $X^{\prime}$ is the dual of $X$, and $\Phi$ is bounded on each bounded subset of $X ; \Psi: X \rightarrow \mathbb{R}$ is a continuously Gâteaux differentiable functional whose Gâteaux derivative is compact; $I \subseteq \mathbb{R}$ is an interval. Assume that

$$
\lim _{\|u\| \rightarrow+\infty}(\Phi(x)+\lambda \Psi(x))=+\infty
$$

for all $\lambda \in I$, and that there exists $h \in \mathbb{R}$ such that

$$
\sup _{\lambda \in I} \inf _{x \in X}(\Phi(x)+\lambda(\Psi(x)+h))<\inf _{x \in X} \sup _{\lambda \in I}(\Phi(x)+\lambda(\Psi(x)+h)) .
$$

Then, there exists an open interval $\Lambda \subseteq I$ and a positive real number $\rho$ with the following property: for each $\lambda \in \Lambda$ and every $C^{1}$ functional $J: X \mapsto \mathbb{R}$ with compact derivative, there exists $\delta>0$ such that, for each $\mu \in[0, \delta]$ the equation

$$
\Phi^{\prime}(x)+\lambda \Psi^{\prime}(x)+\mu J^{\prime}(x)=0
$$

has at least three solutions in $X$ whose norms are less than $\rho$.

Proposition 2.3. [23, Proposition 3.1]Let $X$ be a non-empty set and $\Phi, \Psi$ two real functions on $X$. Assume that there are $r>0$ and $x_{0}, x_{1} \in X$ such that

$$
\Phi\left(x_{0}\right)=-\Psi\left(x_{0}\right)=0, \quad \Phi\left(x_{1}\right)>r, \quad \sup _{\left.\left.x \in \Phi^{-1}(]-\infty, r\right]\right)}-\Psi(x)<r \frac{-\Psi\left(x_{1}\right)}{\Phi\left(x_{1}\right)} .
$$

Then, for each $h$ satisfying

$$
\sup _{\left.\left.x \in \Phi^{-1}(]-\infty, r\right]\right)}-\Psi(x)<h<r \frac{-\Psi\left(x_{1}\right)}{\Phi\left(x_{1}\right)}
$$

one has

$$
\sup _{\lambda \geq 0} \inf _{x \in X}(\Phi(x)+\lambda(\Psi(x)+h))<\inf _{x \in X} \sup _{\lambda \geq 0}(\Phi(x)+\lambda(\Psi(x)+h)) .
$$

In what follows, $X$ denotes the Sobolev space with variable exponent $W^{1, p(x)}(\Omega)$.

\section{Proof of main result}

The operator $\Phi$ is well defined and of class $C^{1}$ (see [10]). The Fréchet derivative of $\Phi$ is the operator $\Phi^{\prime}: X \mapsto X^{\prime}$ defined as

$$
\left\langle\Phi^{\prime}(u), v\right\rangle=\int_{\Omega}\left(|\nabla u|^{p(x)-2}+|\nabla u|^{q(x)-2}\right) \nabla u \nabla v d x+\int_{\Omega}\left(|u|^{p(x)-2}+|u|^{p(x)-2}\right) u v d x,
$$

for any $u, v \in X$. We start by proving some properties of the operator $\Phi^{\prime}$.

Theorem 3.1. The following statements holds.

(1) $\Phi^{\prime}$ is continuous and strictly monotone;

(2) $\Phi^{\prime}$ is of type $\left(S_{+}\right)$;

(3) $\Phi^{\prime}$ is an homeomorphism. 
Proof of Theorem 3.1. (1) Since $\Phi^{\prime}$ is the Fréchet derivative of $\Phi$, it follows that $\Phi^{\prime}$ is continuous. Using the elementary inequalities [31]

$$
\begin{array}{lll}
|x-y|^{\gamma} \leq 2^{\gamma}\left(\left.|x|\right|^{\gamma-2} x-|y|^{\gamma-2} y\right)(x-y) & \text {,if } \quad \gamma \geq 2, \\
|x-y|^{2} \leq \frac{1}{(\gamma-1)}(|x|+|y|)^{2-\gamma}\left(|x|^{\gamma-2} x-|y|^{\gamma-2} y\right)(x-y) & \text {, if } 1<\gamma<2,
\end{array}
$$

for all $(x, y) \in \mathbb{R}^{N} \times \mathbb{R}^{N}$, we obtain for all $u, v \in X$ such that $u \neq v$,

$$
\begin{aligned}
\left\langle\Phi^{\prime}(u)-\Phi^{\prime}(v), u-v\right\rangle & =\int_{\Omega}\left(|\nabla u|^{p(x)-2} \nabla u-|\nabla v|^{p(x)-2} \nabla v\right)(\nabla u-\nabla v) d x \\
& +\int_{\Omega}\left(|\nabla u|^{q(x)-2} \nabla u-|\nabla v|^{q(x)-2} \nabla v\right)(\nabla u-\nabla v) d x \\
& +\int_{\Omega}\left(|u|^{p(x)-2} u-|v|^{p(x)-2} v\right)(u-v) d x \\
& +\int_{\Omega}\left(|u|^{q(x)-2} u-|v|^{q(x)-2} v\right)(u-v) d x>0,
\end{aligned}
$$

which means that $\Phi^{\prime}$ is strictly monotone.

(2) Let $\left(u_{n}\right)_{n}$ be a sequence of $X$ such that

$u_{n} \rightarrow u$ weakly in $X$ as $n \rightarrow+\infty$ and $\lim \sup \left\langle\Phi^{\prime}\left(u_{n}\right), u_{n}-u\right\rangle \leq 0$. Since $q(x) \leq$ $p(x)<p^{\partial}(x)$, then by Proposition 2.1 the embeddings from $W^{1, p(x)}(\Omega)$ to $L^{p(x)}(\Omega)$ and to $L^{q(x)}(\Omega)$ are compact. So we have

$$
\int_{\Omega}\left|u_{n}\right|^{p(x)-2} u_{n}\left(u_{n}-u\right) d x \rightarrow 0 \text { and } \int_{\Omega}\left|u_{n}\right|^{q(x)-2} u_{n}\left(u_{n}-u\right) d x \rightarrow 0 .
$$

Thus

$$
\int_{\Omega}\left|\nabla u_{k}\right|^{p(x)-2} \nabla u_{k}\left(\nabla u_{k}-\nabla u\right) d x+\int_{\Omega}\left|\nabla u_{k}\right|^{q(x)-2} \nabla u_{k}\left(\nabla u_{k}-\nabla u\right) d x \rightarrow 0 .
$$

Since

$$
\int_{\Omega}\left|\nabla u_{k}\right|^{p(x)-2} \nabla u_{k}\left(\nabla u_{k}-\nabla u\right) d x \text { and } \int_{\Omega}\left|\nabla u_{k}\right|^{q(x)-2} \nabla u_{k}\left(\nabla u_{k}-\nabla u\right) d x
$$

have the same sign, then each term converges to 0 . Consequently

$$
\int_{\Omega}\left|\nabla u_{k}\right|^{p(x)-2} \nabla u_{k}\left(\nabla u_{k}-\nabla u\right) d x \rightarrow 0 .
$$

According to the fact that the mapping $\Delta_{p(x)}$ is of type $\left(S_{+}\right)$(see, [15]). We deduce that $u_{k} \rightarrow u$ strongly in $X$ as $n \rightarrow+\infty$.

(3) Note that the strict monotonicity of $\Phi^{\prime}$ implies its injectivity. To show that $\Phi^{\prime}$ is a surjection, it suffices to show that it is coercive. Indeed, using Proposition 2.2 and since $p^{-}-1>0$, we obtain then for each $u \in X$ such that $\|u\| \geq 1$

$$
\begin{aligned}
\frac{\left\langle\Phi^{\prime}(u), u\right\rangle}{\|u\|} & =\frac{\int_{\Omega}\left[|\nabla u|^{p(x)}+|u|^{p(x)}\right] d x+\int_{\Omega}\left[|\nabla u|^{q(x)}+|u|^{q(x)}\right] d x}{\|u\|} \\
& =\frac{\rho_{p}(u)+\rho_{q}(u)}{\|u\|} \geq \frac{\rho_{p}(u)}{\|u\|} \geq \frac{\|u\| p^{-}}{\|u\|} \geq\|u\|^{p^{-}-1} \rightarrow \infty \quad \text { as } \quad\|u\| \rightarrow \infty .
\end{aligned}
$$


Consequently, the operator $\Phi^{\prime}$ is a surjection, thus $\Phi^{\prime}$ admits an inverse mapping. It remains then to show that $\Phi^{\prime-1}$ is continuous. For that, let $\left(f_{n}\right)_{n}$ be a sequence of $X^{\prime}$ such that $f_{n} \rightarrow f$ in $X^{\prime}$ as $n \rightarrow+\infty$. Let $u_{n}$ and $u$ in $X$ such that $\Phi^{\prime-1}\left(f_{n}\right)=u_{n}$ and $\Phi^{\prime-1}(f)=u$. By the coercivity of $\Phi^{\prime}$, one deduce that the sequence $\left(u_{n}\right)$ is bounded in $X$. As $X$ is reflexive (Proposition 2.1), for a subsequence still denoted $\left(u_{n}\right)$, we have $u_{n} \rightarrow \hat{u}$ weakly in $X$ as $n \rightarrow+\infty$, which implies

$$
\lim _{n \rightarrow+\infty}\left\langle\Phi^{\prime}\left(u_{n}\right)-\Phi^{\prime}(u), u_{n}-\hat{u}\right\rangle=\lim _{n \rightarrow+\infty}\left\langle f_{n}-f, u_{n}-\hat{u}\right\rangle=0 .
$$

It follows by the property $\left(S_{+}\right)$and by the continuity of $\Phi^{\prime}$ that $u_{n} \mapsto \hat{u}$ strongly in $X$ and $\Phi^{\prime}\left(u_{n}\right) \mapsto \Phi^{\prime}(\hat{u})=\Phi^{\prime}(u)$ in $X^{\prime}$ as $n \mapsto+\infty$. Moreover, since $\Phi^{\prime}$ is an injection, we conclude that $\hat{u}=u$. This completes the proof.

We are now in a position to give the proof of our main result.

Proof of Theorem 1.1. Set $\Phi(u), \Psi(u)$ and $J(u)$ as in (1.4), (1.5) and (1.6). For each $u, v \in X$, one has

$$
\begin{gathered}
\left\langle\Phi^{\prime}(u), v\right\rangle=\int_{\Omega}\left(|\nabla u|^{p(x)-2}+|\nabla u|^{q(x)-2}\right) \nabla u \nabla v d x+\int_{\Omega}\left(|u|^{p(x)-2}+|u|^{q(x)-2}\right) u v d x \\
\left\langle\Psi^{\prime}(u), v\right\rangle=-\int_{\Omega} f(x, u) v d x \\
\left\langle J^{\prime}(u), v\right\rangle=-\int_{\partial \Omega} g(x, u) v d x .
\end{gathered}
$$

From Theorem 3.1 and [10, Proposition 4], the functional $\Phi$ is a continuous Gâteaux differentiable and sequentially weakly lower semicontinuous functional whose Gâteaux derivative admits a continuous inverse on $X^{\prime}$. By $(H 1)$ and $(H 5), \Psi$ and $J$ are continuously Gâteaux differentiable functionals. Moreover, using the compacity of the embedding $W^{1, p(x)}(\Omega) \hookrightarrow L^{p(x)}(\Omega)$ and the trace embedding $W^{1, p(x)}(\Omega) \hookrightarrow L^{p(x)}(\partial \Omega)$ (Proposition 2.1), we deduce that $\Psi^{\prime}$ and $J^{\prime}$ are compacts. Obviously, $\Phi$ is bounded on each bounded subset of $X$ under our assumptions. If $\|u\| \geq 1$, then

$$
\begin{aligned}
\Phi(u) & =\int_{\Omega}\left(\frac{|\nabla u|^{p(x)}}{p(x)}+\frac{|\nabla u|^{q(x)}}{q(x)}\right) d x+\int_{\Omega}\left(\frac{|u|^{p(x)}}{p(x)}+\frac{|u|^{q(x)}}{q(x)}\right) d x \\
& \geq \frac{1}{p^{+}} \rho_{p}(u)+\frac{1}{q^{+}} \rho_{q}(u) \\
& \geq \frac{1}{p^{+}} \rho_{p}(u) \\
& \geq \frac{1}{p^{+}}\|u\|^{p^{-}} .
\end{aligned}
$$


Meanwhile, using (H4), we obtain, for each $\lambda \in \Lambda \subseteq[0,+\infty)$ and any $u \in X$, that

$$
\begin{aligned}
\lambda \Psi(u) & =-\lambda \int_{\Omega} F(x, u) d x \\
& \geq-\lambda \int_{\Omega} c_{2}\left(1+|u|^{\gamma(x)}\right) d x \\
& \geq-\lambda c_{2}\left(|\Omega|+\|u\|_{\gamma(x)}^{\gamma^{+}}\right) \\
& \geq-c_{2}^{\prime}\left(1+\|u\|_{\gamma(x)}^{\gamma^{+}}\right) \\
& \geq-c_{2}^{\prime \prime}\left(1+\|u\|^{\gamma^{+}}\right),
\end{aligned}
$$

where $c_{2}^{\prime}$ and $c_{2}^{\prime \prime}$ are positive constants and $\|\cdot\|_{\gamma(x)}$ is the usual norm of $W^{1, \gamma(x)}(\Omega)$. Combining the above two inequalities, we obtain

$$
\Phi(u)+\lambda \Psi(u) \geq \frac{1}{p^{+}}\|u\|^{p^{-}}-c^{\prime \prime}{ }_{2}\left(1+\|u\|^{\gamma^{+}}\right),
$$

since $\gamma^{+}<p^{-}$, it follows that

$$
\lim _{\|u\| \rightarrow+\infty} \Phi(u)+\lambda \Psi(u)=+\infty \quad \forall u \in X, \quad \lambda \in[0,+\infty) .
$$

Then assumption (2.1) of Theorem 2.1 is satisfied.

Next, we will prove that assumption (2.2) is also satisfied. To do that, it suffices to verify the conditions of Proposition 2.3. Let $u_{0}=0$. By the definition of $F$, we can easily have

$$
\Phi\left(u_{0}\right)=-\Psi\left(u_{0}\right)=0 .
$$

Now we claim that (2.2) is satisfied.

From $(H 3)$, there exists $\eta \in[0,1], c_{3}>0$, such that

$$
F(x, s)<c_{3}|s|^{p_{1}(x)}<c_{3}|s|^{p_{1}^{-}} \quad \forall s \in[-\eta, \eta] \text {, a.e } x \in \Omega \text {. }
$$

Then, from (H4), we can find a constant $M>0$ such that $F(x, s)<M|s|^{p_{1}^{-}}$, for all $s \in \mathbb{R}$ and a.e $x \in \Omega$. Consequently, by the Sobolev embedding theorem, $W^{1, p(x)}(\Omega) \hookrightarrow L^{p_{1}^{-}}(\Omega)$ is continuous. For suitable positive constants $c_{4}, c_{5}$, we have

$$
-\Psi(u)=\int_{\Omega} F(x, u) d x<M \int_{\Omega}|u|^{p_{1}^{-}} d x \leq c_{4}\|u\|^{p_{1}^{-}} \leq c_{5} r^{p_{1}^{-} / p^{+}}
$$

when $\|u\|^{p^{+}} / p^{+}=\frac{\|u\|^{p^{+}}}{p^{+}} \leq r$. Hence, being $p_{1}^{-}>p^{+}$, it follows that

$$
\lim _{r \rightarrow 0^{+}} \frac{\sup _{\|u\| p^{+} / p^{+} \leq r}(-\Psi(u))}{r}=0 .
$$

Let $u_{1} \in C^{1}(\Omega)$ be a positive function in $\Omega$, with $\max _{\bar{\Omega}} u_{1} \leq c_{1}$. Then, $u_{1} \in X$ and $\Phi\left(u_{1}\right)>0$. In view of (H2), we also have $-\Psi\left(u_{1}\right)=\int_{\Omega} F\left(x, u_{1}\right) d x>0$. Therefore, from (3.1), we can find 
$r \in\left(0, \min \left\{\Phi\left(u_{1}\right), \frac{1}{p^{+}}\right\}\right)$such that

$$
\sup _{\|u\|^{p^{+} / p^{+} \leq r}}(-\Psi(u))<r \frac{-\Psi\left(u_{1}\right)}{\Phi\left(u_{1}\right)} .
$$

Now, let $u \in \Phi^{-1}((-\infty, r])$. Since $q(x) \leq p(x)$ and $p(x)<p^{+}$, it follows that $\rho_{p}(u)+\rho_{q}(u) \leq$ $p^{+} r$. Then $\rho_{p}(u) \leq p^{+} r<1$ which, by Proposition 2.2, implies $\|u\|<1$. Consequently we have

$$
\frac{1}{p^{+}}\|u\|^{p^{+}} \leq \frac{1}{p^{+}} \rho_{p}(u) \leq \frac{1}{p^{+}} \int_{\Omega}\left(|\nabla u|^{p(x)}+|u|^{p(x)}\right) d x<r .
$$

Therefore, we infer that $\Phi^{-1}((-\infty, r]) \subset\left\{u \in X: \frac{1}{p^{+}}\|u\|^{p^{+}}<r\right\}$, and so

$$
\sup _{u \in \Phi^{-1}((-\infty, r])}(-\Psi(u))<r \frac{-\Psi\left(u_{1}\right)}{\Phi\left(u_{1}\right)} .
$$

At this point, conclusion follows from Proposition 2.3 and Theorem 2.1.

\section{Conclusion}

In this paper, we have established the existence of solutions for Steklov problem with $(p(x), q(x))$-Laplacian operator via Ricceri's three critical points theorem. Our contribution is not only new in the given setting, but also generalizes special cases of previous works involving $p$ and $p(x)$-Laplacian operators.

\section{Acknowledgements}

The authors would like to thank the referees for their valuable suggestions and comments which helped us to improve our manuscript.

\section{References}

[1] E. Acerbi and G. Mingione, Regularity results for a class of functionals with nonstandard growth, Arch. Ration. Mech. Anal. 156 (2001), no. 2, 121-140.

[2] G. A. Afrouzi,Three solutions for a Dirichlet boundary value problem involving the p-Laplacian. Nonlinear Anal. 66(2007), 2281-2288.

[3] S. N.Antontsev and J. F. Rodrigues, On stationary thermorheological viscous flows, Ann. Univ. Ferrara Sez. VII Sci. Mat. 52 (2006) 19-36.

[4] M. Bocea and M. Mihăilescu: Г-convergence of power-law functionals with variable exponents, Nonlinear Analysis 73 (2010), 110-121.

[5] M. Bocea, M. Mihăilescu, and C. Popovici: On the asymptotic behavior of variable exponent power-law functionals and applications, Ricerche di Matematica 59 (2010), 207-238.

[6] M. Bocea, M. Mihăilescu, M. Pérez-Llanos, and J. D. Rossi: Models for growth of heterogeneous sandpiles via Mosco convergence, Asymptotic Analysis 78 (2012), 11-36.

[7] G. Bonanno, P. Candito, Three solutions to a Neumann problem for elliptic equations involving the p-Laplacian. Arch. Math. (Basel), 80(2003), 424-429.

[8] G. Bonanno, R. Livrea, Multiplicity theorems for the Dirichlet problem involving the p-Laplacian. Nonlinear Anal. 54(2003), 1-7. 
[9] G. Bonanno, G. Molica Bisci, V. Rădulescu, Multiple solutions of generalized Yamabe equations on Riemannian manifolds and applications to Emden-Fowler problems. Nonlinear Anal. Real World Appl. 12(2011), 2656-2665.

[10] M. M. Boureanu, D. N. Udrea, Existence and multiplicity results for elliptic problems with $p($.$) -Growth conditions.$ Nonlinear Anal. Real World Appl. 14(2013), 1829-1844.

[11] Y. Chen, S. Levine and R. Ran, Variable exponent, linear growth functionals in image restoration, SIAM J. Appl. Math. 66 (2006), 1383-1406.

[12] S. G. Deng, Positive solutions for Robin problem involving the $p(x)$-Laplacian. J. Math. Anal. Appl. 360(2009), 548-560.

[13] L. Diening, P. Harjulehto, P. Hästö, M. Ruz̃ic̃ka, Lebesgue and Sobolev spaces with variable exponents, Lecture Notes in Mathematics, vol. 2017, Springer-Verlag, Berlin, 2011.

[14] X. L. Fan, X. Y. Han, Existence and multiplicity of solutions for $p(x)$-Laplacian equations in $\mathbb{R}^{N}$. Nonlinear Anal. 59(2004), 173-188.

[15] X. L. Fan, Q. H. Zhang, Existence of solutions for $p(x)$-Laplacian Dirichlet problems. Nonlinear Anal. 52(2003), 1843-1852.

[16] X. L. Fan, D. Zhao, On the spaces $L^{p(x)}(\Omega)$ and $W^{m, p(x)}(\Omega)$. J. Math. Anal. App. 263(2001), 424-446.

[17] G. Fragnelli, Positive periodic solutions for a system of anisotropic parabolic equations, J. Math. Anal. Appl. 73 (2010), 110-121.

[18] O. Kovácik, J. Rákosnik, On spaces $L^{p(x)}$ and $W^{k, p(x)}$. Czechoslovak Math. J. 41(1991), 592-618.

[19] Q. Liu, Existence of three solutions for $p(x)$-Laplacian equations. Nonlinear Anal. 68(2008), 2119-2127.

[20] M. Mihăilescu, Existence and multiplicity of solutions for a Neumann problem involving the $p(x)$-Laplace operator. Nonlinear Anal. 67(2007), 1419-1425.

[21] W. Orlicz, Über konjugierte Exponentenfolgen, Studia Math., 3 (1931), 200-211

[22] K.R. Rajagopal and M. Ruz̃ic̃ka: Mathematical modeling of electrorheological materials, Contin. Mech. Thermodyn. 13 (2001), 59-78.

[23] B. Ricceri, Existence of three solutions fo a class of elliptic eigenvalue problems. Math. Comput. Modelling. 32(2000), 1485-1494.

[24] B. Ricceri, A three critical points theorem revisited. Nonlinear Anal. 70(2009), 3084-3089.

[25] M. Ruz̃ic̃ka: Flow of shear dependent electrorheological fuids: unsteady space periodic case, Applied Nonlinear Analysis, 485-504, Kluwer/Plenum, New York, 1999.

[26] M. Ruz̃ic̃ka:Electrorheological fuids: modeling and mathematical theory, Lecture Notes in Mathematics 1748, Springer-Verlag, Berlin, 2000.

[27] K. Saoudi, Existence and multiplicity of solutions for a quasilinear equation involving the $p(x)$-Laplace operator, Complex Variables and Elliptic Equations, 62 (3), 318-332 (2017).

[28] K Saoudi, A Ghanmi, A multiplicity results for a singular equation involving the $p(x)$-Laplace operator, Complex Variables and Elliptic Equations 62 (5), 695-725 (2017).

[29] K. Saoudi, M. Kratou, and S. Alsadhan, Multiplicity results for the Laplacian equation with singular nonlinearities and nonlinear Neumann Boundary Condition, Int. J. Differ. Equ. 2016 (Article ID 3149482), 15 (2016).

[30] X. Shi, X. Ding, Existence and multiplicity of solutions for a general $p(x)$-Laplacian Neumann problem. Nonlinear Anal. 70(2009), 3715-3720.

[31] J. Simon, Régularité de la solution d'une équation non linéaire dans $\mathbb{R}^{N}$. Vol.665 of Lecture Notes in Math., Springer, Berlin, (1978), 205-227.

[32] A. Zerouali, B. Karim, O. Chakrone, A. Anane, Existence and Multiplicity results for elliptic problems with Nonlinear Boundary conditions and variable exponents. Bol. Soc Paran. Mat. (3s)v. 332 (2015), 123-133.

[33] V. V. Zhikov: Averaging of functionals of the calculus of variations and elasticity theory, Izv. Akad. Nauk SSSR Ser. Mat., 50 (1986), 675-710. 